\title{
SEPARAÇÃO DE TEMPERATURA E EMISSIVIDADE A PARTIR DE IMAGENS DO INFRAVERMELHO TERMAL: ANÁLISE DE SUAS APLICAÇÕES/RESTRIÇÕES
}

\author{
Separation of Temperature and Emissivity from Thermal Infrared Images: \\ Analysis of their Application/Restriction
}

Atilio Grondona ${ }^{1,2}$

Silvia Beatriz Alves Rolim ${ }^{1}$

1 Universidade Federal do Rio Grande do Sul (UFRGS) - Centro Estadual de Pesquisas em Sensoriamento Remoto e Meteorologia (CEPSRM)Av. Bento Gonçalves, 9.500 - 91501-970. Caixa Postal 15.044 Porto Alegre, RS/Brasil\{atilio.grondona; silvia.rolim \}@ufrgs.br

${ }^{2}$ Faculdade de Tecnologia TecBrasil (FTEC).Rua Comendador Manuel Pereira, 249 - 90030-030.

Porto Alegre, RS/Brasil

\section{Resumo:}

O infravermelho termal (TIR - ThermalInfraRed) é uma porção do espectro eletromagnético com várias aplicações no Sensoriamento Remoto, tais como: geologia, climatologia, análises de processos biológicos, análises geofísicos, avaliação de desastres e detecção de mudanças, entre outras. No TIR a emissão de radiação dos alvos é dominante, comparado com a reflexão, e esta radiação é uma função de duas variáveis, a emissividade e a temperatura do alvo. Para estudos no TIR é necessário estimar com precisão a temperatura e/ou a emissividade a partir da radiação medida, e isto é um problema devido a relação não linear existente entre estas variáveis e a radiação medida. Por isso, nos últimos 40 anos vários pesquisadores têm desenvolvido métodos visando minimizar este problema, porém, todos estes métodos possuem restrições em suas aplicações. Assim, este trabalho tem como objetivo revisar os principais métodos propostosna literaturafacilitando a sua compreensão, reprodução, além de criar umasínteseque permitaráo leitor a escolha do método mais adequado a determinadas situações.

Palavras-chave: TIR, TES, cálculo da temperatura, emissividade

\begin{abstract}
:
Remote Sensing data coming from the Thermal InfraRed (TIR) channel of electromagnetic spectrum has several applications in remote sensing, geology, climatology, biological process analysis, geophysical process analysis, disaster assessment and change detection analysis. In the TIR region, the emission of the targets is dominant when compared with reflection. This radiation is a function of two unknowns - the emissivity and the temperature of the target. To study TIR, it is necessary estimate the temperature and/or emissivity from de measured radiation with precision. This process is usually difficult due to a non-linear relationship between these two unknowns and the measured radiation. Therefore, in the last 40 years, several researchers have developed approaches to minimize this problem. However, all these methods have constraints in their applications. This study try to review the main methods available in the literature, facilitating their understanding and replication and creating a summary that allows the reader to choose the most suitable method in their own application.
\end{abstract}

Keywords: TIR, TES, temperature measurement, emissivity 


\section{Introdução}

Os dados de radiância do infravermelho termal (TIR -Thermal InfraRed) podem ser utilizados para calcular a temperatura e a emissividade dos alvos, e possuemaplicações em diversas áreas, tais como: geologia, climatologia, análises de processos biológicos, análisesgeofísicas, estudos da pluma atmosférica, identificação de constituintes da superfície do solo e para o mapeamento do uso da terra, avaliação de desastres, detecção de poluição e detecção de mudanças, entre outras (Collins et al., 1999; Collins et al., 2001). Quando se utiliza o TIR com tal objetivo é importante extrair os dados de temperatura e emissividade coerentes, tal que combinados formam o espectro de radiância do alvo.

Sendo assim, nos últimos 40 anos alguns autores como Collins et al. (1999) e (2001); Hook et al. (1992); Kealy e Hook (1993); Gillespie (1985), Gillespie' (1992); Gillespie ${ }^{2}$ (1992); Gillespie et al. (1999); Watson ${ }^{1}$ (1992) e Watson ${ }^{2}$ (1992); Schmugge et al. (1998); Coll et al.(2007); Barducci e Pipi (1996); Kahle e Alley (1992) entre outros vêm pesquisando, desenvolvendo, e melhorando os métodos de separação da temperatura e emissividade (TES -Temperature and Emissivity Separation) para o TIR.

No TIR a grandeza física medida remotamente pelo sensor é a radiância espectral, definida como o fluxo radiante numa dada direção, a partir de uma superfície normalizada com respeito à área da superfície e unidade de ângulo sólido (Siegal et al., 1982). Isto é válido para todo o espectro eletromagnético, porém para o TIR existe ainda a emissividade espectral $(\varepsilon)$, que é um índice que informa o quão capaz um material é de transformar a energia que incide sobre ele em radiação térmica. Este índice está relacionado à radiância espectral e a função de Planck para um corpo negro (sob o mesmo comprimento de onda e temperatura). A função de Planck é dada pela seguinte equação:

$$
B(\lambda, T)=\frac{C_{1}}{\lambda^{5} \pi\left(e^{C_{2} / \lambda T}-1\right)} \quad\left(W / m^{2} s r \mu m\right)
$$

onde $B(\lambda, T)$ é a radiação reemitida pelo corpo negro, $\lambda$ é o comprimento de onda $(\mu m), T$ é a temperatura da superfície $(K), C_{1}$ é a primeira constante de radiação $\left(3,74151 \times 10^{-16} W^{2}\right)$ e $C_{2}$ é a segunda constante de radiação $(0,0143879 m K)$.

Se a superfície terrestre fosse um corpo negro perfeito a uma temperatura constante e sem a intervenção da atmosfera, a radiância medida no sensor seria dada pela equação 1 . Os alvos não emitem radiância como um corpo negro, e parte da energia absorvida é dissipada em forma de energia térmica. A parte de energia absorvida que um corpo dissipa como energia térmica pode ser calculada como:

$$
\varepsilon(\lambda, T)=\frac{I(\lambda, T)}{B(\lambda, T)}
$$

onde $I(\lambda, T)$ é a radiância medida no sensor no comprimento de onda $\lambda$ e temperatura $T$ desconsiderando os efeitos da atmosfera. A equação 2 é uma razão entre a radiância de um dado material e a radiância de um corpo negro sob mesma temperatura e comprimento de onda. Logo, para qualquer material real e conhecendo a emissividade e a temperatura do alvo, a partir da equação 2 a radiância medida no sensor pode ser escrita como: 


$$
I(\lambda, T)=\varepsilon(\lambda, T) B(\lambda, T)
$$

Assim, utilizando a equação 1, a equação 3 pode-se expressa como:

$$
I(\lambda, T)=\varepsilon(\lambda, T) \frac{C_{1}}{\lambda^{5} \pi\left(e^{C_{2} / \lambda T}-1\right)}
$$

$\mathrm{Na}$ equação 4 é visível a existência de uma relação não linear entre a temperatura $(T)$ o comprimento de onda $(\lambda)$ e a radiância medida no sensor $I(\lambda, T)$. Esta não linearidade contribui para multiplicar os efeitos do espalhamento atmosférico, efeitos emissivos em cenas com geometrias mais complexas, cenas com heterogeneidade da atmosfera, como por exemplo, cobertura parcial de nuvens, e ainda, cenas com superfícies adjacentes diferentes, como árvores e arbustos (Collins et al., 1999 e 2001).

Devido a presença da atmosfera a radiância que atinge o sensor deve, na maioria das vezes, ser corrigida para os efeitos de emissão, atenuação e espalhamento atmosférico antes da aplicação de alguns dos métodos abordados neste trabalho. Na literatura a radiação medida no sensor incluindo a contribuição da atmosfera é:

$$
\begin{gathered}
I(\lambda, T)=R(\lambda, T) \tau_{\lambda}+R_{\lambda}^{\uparrow} \\
R(\lambda, T)=\varepsilon(\lambda, T) B(\lambda, T)+(1-\varepsilon(\lambda, T)) R_{\lambda}^{\downarrow}
\end{gathered}
$$

onde $R^{\downarrow}(\lambda, T)$ é a radiância espectral descendente que incide sobre a superfície da terra proveniente do espalhamento e da emissão atmosférica, $R^{\uparrow}(\lambda, T)$ é radiância espectral ascendente da atmosfera que atinge o sensor, $\boldsymbol{\tau}_{\lambda}$ é transmissividade espectral da atmosfera e $R(\lambda, T)$ é a radiância medida no solo.

Neste trabalho serão abordados os principais métodos utilizados pela comunidade científica/acadêmica, muitos dos quais serviram como base para métodos mais novos e complexos. Estes métodos são os de maior aplicabilidade, ou seja, exigem menos restrições quanto ao tipo de sensor, alvo, número de imagens e número de bandas espectrais. Existem ainda outros métodos, porém,estes se aplicam a casos mais específicos e não serão abordados nesta investigação.O grande número de métodos existentes e os problemas encontrados no TIR serviram como motivação para este trabalho, que tem como objetivo revisar e discutir os principais métodos desenvolvendo,portanto, um guia para facilitar o entendimento, reprodução e escolha do método mais adequado a ser aplicado.

\section{Problemas na Separação Temperatura/Emissividade}

O problema na separação da temperatura e emissividade é complexo devido à relação não linear existente entre a temperatura e a radiância (Li et al., 1999; Collins et al., 2001). A radiância pode ser calculada utilizando a função de Planck e a emissividade do alvo (para determinado comprimento de onda e temperatura). Independente do número de bandas espectrais utilizadas para as medidas de radiância, sempre existirá uma variável a mais do que as medidas realizadas. Por exemplo, um radiômetro com $N$ bandas espectrais possui $N$ medidas de radiância e $N+1$ variáveis ( $N$ emissividades para cada banda e uma temperatura $T$ ). Isto torna o sistema 
indeterminado, uma vez que ele possui mais variáveis que possíveis equações, a menos que restrições complementares sejam incluídas ao sistema.

Deve-se salientar que outro problema no TIR é a atmosfera, principalmente em ambientes úmidos, onde a grande umidade interfere na aquisição correta dos dados da superfície terrestre. Assim, sempre que possível é necessário realizar a correção atmosférica, já que devido a não linearidade do TIR o erro propagado no cálculo da temperatura/emissividade pode ser significativo. No entanto, para tal correção dados auxiliares são necessários, e muitas vezes não estão disponíveis, assim a correção atmosférica se torna um problema adicional principalmente se forem utilizados modelos atmosféricos não condizentes com a área estudada.

\section{Métodos de Separação de Temperatura e Emissividade}

Diversos métodos de separação de temperatura e emissividade foram desenvolvidos nas últimas décadas e muitos outros estão atualmente em estudo, todos têm o objetivo de separar informações de temperatura e emissividade. Porém, existe um problema comum a todos os métodos, que é de haver mais variáveis que equações. Assim, visando mitigar este problema, cada método é desenvolvido para ser aplicado em condições específicas, e devem satisfazer um conjunto de hipóteses para que o resultado obtido seja confiável. Com tais restrições é evidente que o melhor resultado está diretamente ligado a escolha do método que satisfaça todas as hipóteses e condições, ou ao método que mais as cumpre, ou seja, que viola a menor quantidade destas hipóteses e condições. Estes métodos podem ser divididos, basicamente, em três grandes grupos (Gillespie $\left.{ }^{1}, 1992\right)$ : realce de temperatura e dados composicionais simultaneamente (Razão Espectral), realce de temperatura e dados composicionais individualmente (Métodos de Duas Temperaturas, Índices Espectrais Independentes da Temperatura, Método de Renormalização da Emissividade, Método da banda de Referência, Método da Emissividade Normalizada, Método dos Resíduos Alpha, Método da Máxima-Mínima Diferença, Método do Corpo Cinza) e realces híbridos (Realce de Contraste por Decorrelação). Outra definição importante, que também pode ser usada para subdividir os métodos, é com relação aos métodos que calculam a emissividade relativa e os que calculam a emissividade absoluta. $O$ termo "emissividade relativa" é usado quando o valor da emissividade é calculado com relação a uma emissividade de referência, e neste caso o resultado é a forma da curva espectral da emissividade e não o seu valor em si. O termo "emissividade absoluta" é referente ao valor absoluto desta grandeza. A emissividade absoluta é muito importante para estimativas da temperatura de superfície (Sobrino e Jimenez-Muñoz, 2002).

\subsection{Realce de Contraste por Decorrelação (RCD)}

O realce de contraste por decorrelação não gera um resultado quantitativo. $\mathrm{O}$ resultado final da aplicação deste método é a obtenção de um conjunto de imagens das quais se podem formar composições falsa-cor e extrair informações visuais das variações na temperatura e emissividade. Diferentes alvos e diferenças na composição dos alvos implicam em diferenças na emissividade, mesmo sob condições isotermais. No entanto, esta variação não é representativa e faz com que a variabilidade e o contraste da emissividade no TIR sejam baixos, resultando em uma alta correlação para as diferentes bandas. Um dos métodos utilizados para contornar este problema é baseado na transformação por Componentes Principais (CP), e também é conhecido como realce de contraste por decorrelação (Soha e Schwartz, 1978; Kahle e Rowan, 1980). Como este 
método é baseado nas PC pode ser facilmente aplicado a imagens com qualquer número de bandas, e pode ser resumido em quatro passos:

$1^{\circ}$ ) Calcular a matriz de covariância da imagem e os respectivos autovalores;

$2^{\circ}$ ) A imagem é transformada do domínio da radiância para o espaço das PC's, e os dados transformados têm a propriedade de serem decorrelacionados;

$3^{\circ}$ ) As PC's mais correlacionadas têm seus contrastes realçados separadamente;

$4^{\circ}$ ) A transformada inversa é aplicada, as PC's voltam ao espaço da radiância;

Em geral, as matizes antes e depois da transformação são muito similares enquanto as saturações são aumentadas, deste modo, uma composição falsa-cor (RGB) destas imagens mostra diferenças de emissividade como variações nas cores, enquanto que diferenças na temperatura são mostradas como variações na intensidade.

\subsection{Razão Espectral (RE)}

As razões entre as radiâncias são menos sensíveis a pequenas variações na temperatura, ao contrário das radiâncias em si (Watson ${ }^{1}$, 1992). Assim, somente um valor aproximado da temperatura é necessário para determinar a razão de emissividade com grande precisão.

Se a radiância é medida em $N$ bandas espectrais, então o problema se torna um sistema composto por $N$ equações, uma para cada emissividade mais a temperatura $T$, ou seja, o sistema possui mais variáveis $(N+1)$ que equações. Para encontrar uma solução para este sistema é necessário estimar a temperatura $T$, ou eliminar a dependência do sistema desta variável. Neste método é proposto utilizar razões espectrais entre bandas adjacentes. As razões espectrais são eficientes em detectar variações na emissividade, porém, possuem limitações para a análise sobre todo o espectro, e uma dessas limitações é o uso de uma emissividade absoluta para a solução do sistema. Inicialmente, a presença da atmosfera será desconsiderada (equação 3) e a razão de radiância é dada por:

$$
\frac{R_{i+1}\left(\lambda_{i+1}, T\right)}{R_{i}\left(\lambda_{i}, T\right)}=\left(\frac{\varepsilon_{i+1}\left(\lambda_{i+1}, T\right)}{\varepsilon_{i}\left(\lambda_{i}, T\right)}\right)\left(\frac{B_{i+1}\left(\lambda_{i+1}, T\right)}{B_{i}\left(\lambda_{i}, T\right)}\right)
$$

com $i=1, \ldots, N-1$.

Aparentemente, o problema de haver mais variáveis que equações perdura, no entanto, a razão $B_{i+1}\left(\lambda_{i+1}, T\right) / B_{i}\left(\lambda_{i}, T\right)$ é muito menos sensível a pequenas variações na temperatura do que somente o termo $B_{i}\left(\lambda_{i}, T\right)$ (para o intervalo de temperaturas da terra e nos comprimentos de onda entre 8-14 $\mu \mathrm{m})$. Para o caso específico do sensor ASTER, a razão $B_{i+1}\left(\lambda_{i+1}, T\right) / B_{i}\left(\lambda_{i+1}, T\right)$, na equação 7 , pode ser calculada utilizando uma estimativa da temperatura $(\bar{T})$, e o erro da razão para valores nominais $\bar{T}=300 \mathrm{~K}$ e $\lambda_{i}=10 \mu \mathrm{m}$ é menor que $1 \%$ (Siegel e Howel, 1982; Grondona et al., 2013).

Existem várias formas para estimar a temperatura a partir da radiância termal, no entanto,um método independente do conhecimento prévio da geologia da área consiste em inverter a equação 3 para calcular a temperatura de brilho $\left(T_{\lambda}^{\text {brilho }}\right)$ :

$$
T_{\lambda}^{\text {brilho }}=B_{\lambda}^{-1}\left(\frac{R_{\lambda}}{\varepsilon_{\lambda}}\right)
$$


tal que $T_{\lambda}^{\text {brilho }} \leq T$, já que $\varepsilon_{\lambda} \leq 1$. Desta forma $T_{\lambda}^{\text {brilho }} \leq \max \left(T_{\lambda}^{\text {brilho }}\right) \leq T$, onde $\max \left(T_{\lambda}^{\text {brilho }}\right)$ representa a melhor estimativa para a temperatura de superfície.

Considerando a contribuição da atmosfera,dado pelas equações 5 e 6 , temos 3 possíveis casos:

CASO 1 - Desprezando $R^{\uparrow}(\lambda)$ e para um par de bandas $\left(\lambda_{1}, \lambda_{2}\right)$ a razão é:

$$
\frac{I_{\lambda_{1}}}{I_{\lambda_{2}}}=\left(\frac{\tau_{\lambda_{1}}}{\tau_{\lambda_{2}}}\right)\left(\frac{\varepsilon_{\lambda_{1}} B_{\lambda_{1}}(T)+\left(1-\varepsilon_{\lambda_{1}}\right) R_{\lambda_{1}}^{\downarrow}}{\varepsilon_{\lambda_{2}} B_{\lambda_{2}}(T)+\left(1-\varepsilon_{\lambda_{2}}\right) R_{\lambda_{2}}^{\downarrow}}\right)
$$

e, desde que $B_{\lambda}(T)>>R_{\lambda}^{\downarrow}$, o que é valido para dados diurnos, já que a atmosfera é parcialmente transparente nesta região espectral, segue que

$$
\frac{I_{\lambda_{1}}}{I_{\lambda_{2}}} \approx=\left(\frac{\tau_{\lambda_{1}}}{\tau_{\lambda_{2}}}\right)\left(\frac{\varepsilon_{\lambda_{1}}}{\varepsilon_{\lambda_{2}}}\right)\left(\frac{B_{\lambda_{1}}(T)}{B_{\lambda_{2}}(T)}\right)(1+\omega)
$$

onde $\omega$ pode ser desprezado.

CASO 2 - Incluindo o $R^{\uparrow}(\lambda)$, se existe uma área grande o suficiente que sirva para calibração, como um corpo d'água por exemplo, a radiância ascendente pode ser estimada, e a radiância medida corrigida. Um erro residual da radiância espectral ascendente $\left(\Delta R^{\uparrow}(\lambda)\right)$ introduz um termo secundário no último parêntese da equação 10 com a forma $\Delta R^{\uparrow}(\lambda) / \tau_{\lambda} \varepsilon_{\lambda} B_{\lambda}(T)$. Para dados adquiridos em nível sub-orbital esse erro é menor ou igual a $\omega$ na equação 10.

$$
\Delta R^{\uparrow}(\lambda) / \tau_{\lambda} \varepsilon_{\lambda} B_{\lambda}(T)
$$

Para dados adquiridos em nível sub-orbital esse erro é menor ou igual a $\omega$ na equação 10.

CASO3 - Com correção atmosférica completa, quando os parâmetros atmosféricos na equação 6 podem ser estimados, a razão da radiância corrigida é dada por:

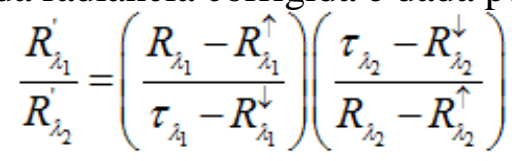

enquanto a razão de emissividade é dada por:

$$
\frac{\varepsilon_{\lambda_{1}}}{\varepsilon_{h_{2}}}=\frac{\left(R_{\lambda_{1}}^{\prime} / R_{h_{2}^{\prime}}^{\prime}\right)\left(B_{h_{2}}(\bar{T})-R_{h_{2}}^{\downarrow}\right)}{B_{\lambda_{1}}(\bar{T})-R_{h_{1}}^{\downarrow}}
$$

\subsection{Método de Duas Temperaturas (MDT)}

Este método (Watson², 1992) supõe que a emissividade é invariante no tempo, ou seja, não ocorrem mudanças significativas na emissividade durante o período de tempo entre as medições. Assim, se for possível observar um alvo em duas temperaturas diferentes também é possível determinar a emissividade espectral do alvo em questão. Esta hipótese é aceitável para alvos 
como rochas e solos secos, mas não está bem estabelecida para a vegetação e solos em que haja mudança na composição durante o intervalo entre as observações. A vantagem deste método é que ele não necessita de nenhum conhecimento a priori sobre a forma da curva espectral do alvo. As observações da cena devem, preferencialmente, ser realizadas em dois horários diferentes dentro do ciclo diurno. A orientação para estas observações é que uma deva ser realizada logo após o meio-dia e a outra logo após a meia-noite (temperaturas máximas e mínimas diárias). Utilizando a equação 3, para duas observações temos:

$$
R_{j}\left(\lambda, T_{j}\right)=\varepsilon\left(\lambda, T_{j}\right) B_{j}\left(\lambda, T_{j}\right), \operatorname{com} j=1,2
$$

onde $j$ é o número de observações em diferentes temperaturas. Para $j=2$ a equação 14 é um sistema de $2 N$ equações com $N+2$ variáveis ( $N$ emissividades e 2 temperaturas), e a solução para o sistema é possível quando $N>1$. Como a emissividade é invariante no tempo para determinado comprimento de onda, para eliminar a emissividade pode-se tomar a razão entre as radiâncias:

$$
\frac{R_{2}\left(\lambda, T_{2}\right)}{R_{1}\left(\lambda, T_{1}\right)}=\frac{B_{2}\left(\lambda, T_{2}\right)}{B_{1}\left(\lambda, T_{1}\right)}=\frac{e^{\left(C_{2} / / T_{1}\right)}-1}{e^{\left(C_{2} / \lambda T_{2}\right)}-1}
$$

Esta equação pode ser resolvida, para as duas temperaturas $\left(T_{1}, T_{2}\right)$, tendo solução única se $N=2$, e tendo mais de uma solução se $N>2$, e neste caso é necessário utilizar algum método de aproximação como, por exemplo, mínimos quadrados. Resultados experimentais mostraram que o método mais satisfatório para solucionar este problema é usar uma das equações para expressar uma das temperaturas como função da outra e então resolver a temperatura restante usando aproximações sucessivas.As temperaturas podem ser limitadas usando $\min (\varepsilon(\lambda))<\varepsilon(\lambda)<1$, com $\min (\varepsilon(\lambda))$ sendo alguma estimativa arbitrária para o menor valor de emissividade que possa ocorrer, assim definindo o intervalo em que as temperaturasestão contidas. Uma vez que as temperaturas forem calculadas é fácil determinar o termo $B_{j}\left(\lambda, T_{j}\right)$ e, subseqüentemente, a emissividade.

Considerando a contribuição da atmosfera (equação 5), pode-se utilizar a diferença temporal da radiância para estimar as variáveis atmosféricas, expressa por:

$$
\Delta I_{\lambda} \equiv I_{\lambda, 1}-I_{\lambda, 2}=\tau_{\lambda} \varepsilon_{\lambda}\left[B_{j}\left(\lambda, T_{1}\right)-B_{j}\left(\lambda, T_{2}\right)\right]+O_{\lambda}
$$

onde $O_{\lambda}=\tau_{\lambda}\left(1-\varepsilon_{\lambda}\right)\left[R_{1}^{\uparrow}(\lambda)-R_{2}^{\uparrow}(\lambda)\right]+\left[R_{1}^{\uparrow}(\lambda)-R_{2}^{\uparrow}(\lambda)\right]$ é o termo atmosférico que pode ser estimado, $\boldsymbol{\tau}_{\lambda}$ é considerado temporalmente invariante. Uma regressão linear entre os valores observados de $\Delta I_{\lambda, j}$ e $T_{1}-T_{2}$ é utilizada para calcular o termo $\mathrm{O}_{\lambda}$ dado pelo intercepto.

\section{4 Índice Espectral Independente da Temperatura (TISI)}

Este método foi inicialmente proposto por Becker e Li (1990), e posteriormente corrigido Becker e Li (1995) e Li et al. (1999). Utilizando a equação 5, defina-se a temperatura de brilho da banda $\left(T_{B}\right)$ e a temperatura de brilho da superfície $\left(T_{S}\right)$, respectivamente, como:

$$
I\left(\lambda, T_{B}\right)=B\left(\lambda, T_{B}\right)
$$




$$
R\left(\lambda, T_{s}\right)=B\left(\lambda, T_{S}\right)
$$

Para moderadas variações da temperatura em torno de uma temperatura de referência $\left(T_{0}\right)$, foi mostrado por Hardy et al. (1934) e Slater (1980) que é válida a seguinte aproximação para a função de Planck:

$$
B_{\lambda}(T)=\alpha_{\lambda}\left(T_{0}\right) T^{n_{2}\left(T_{0}\right)}
$$

onde $\alpha_{\lambda}\left(T_{0}\right)$ e $T^{n_{\lambda}\left(T_{0}\right)}$ são constantes referentes a banda $\lambda$ e a temperatura $T_{0}$, e podem ser obtidas utilizando um ajuste por mínimos quadrados para a equação $19 \operatorname{com} T=T_{0}$. As variáveis $n_{\lambda}\left(T_{0}\right)$ e $\alpha_{\lambda}\left(T_{0}\right)$ são, respectivamente dadas por:

$$
n_{\lambda}\left(T_{0}\right)=\frac{C_{2}}{\lambda T_{0}}\left(1+\frac{1}{e^{\left(C_{2} / \lambda T_{0}\right)}-1}\right) \text { e } \alpha_{\lambda}\left(T_{0}\right)=\frac{B_{\lambda}\left(T_{0}\right)}{T_{0}^{n_{\lambda}\left(T_{0}\right)}}
$$

Portanto, a partir das equações 18 e 19, a radiância pode ser expressa como:

$$
R_{\lambda}\left(T_{0}\right) \cong \alpha_{\lambda}\left(T_{0}\right) T_{0}^{n_{2}\left(T_{0}\right)}
$$

Agora é possível re-escrever a equação 18 como:

$$
R_{\lambda}=\alpha_{\lambda}\left(T_{0}\right) T^{n_{\lambda}\left(T_{0}\right)}=\varepsilon_{\lambda} \alpha_{\lambda}\left(T_{0}\right) T^{n_{\lambda}\left(T_{0}\right)}+\left(1-\varepsilon_{\lambda}\right) R_{\lambda}^{\downarrow}
$$

Se para cada radiância o termo $R_{\lambda}^{\downarrow}$ puder ser desprezado, a equação 21 se torna:

$$
\begin{aligned}
& R_{\lambda}=\varepsilon_{\lambda} \alpha_{\lambda}\left(T_{0}\right) T^{n_{\lambda}\left(T_{0}\right)} \\
& T_{0}^{n_{\lambda}\left(T_{0}\right)}=\varepsilon_{\lambda} T_{S}^{n_{2}\left(T_{0}\right)}
\end{aligned}
$$

Assim é possível obter um índice independente da temperatura, para isso são necessárias, no mínimo, duas banda se uma razão ponderada entre as radiâncias com a finalidade de simplificar a temperatura:

$$
\begin{aligned}
& \frac{\left(R_{\lambda}\right)^{a_{2}}}{\left(R_{\lambda+1}\right)^{a_{\lambda+1}}}=\frac{\left[\varepsilon_{\lambda} \alpha_{\lambda}\left(T_{0}\right)\right]^{a_{2}}}{\left[\varepsilon_{\lambda+1} \alpha_{\lambda+1}\left(T_{0}\right)\right]^{a_{\lambda+1}}} \\
& \frac{T_{0}^{a_{2} n_{2}\left(T_{0}\right)}}{T_{0}^{a_{\lambda+1} n_{\lambda+1}\left(T_{0}\right)}}=\frac{\left[\varepsilon_{\lambda}\right]^{a_{\lambda}}}{\left[\varepsilon_{\lambda+1}\right]^{a_{\lambda+1}}}
\end{aligned}
$$

Utilizando essas equações é fácil definir tal índice:

$$
\begin{gathered}
\operatorname{TISI}_{\lambda, \lambda+1}=\frac{\alpha_{\lambda+1}^{n_{\lambda, \lambda+1}}}{\alpha_{\lambda}} \frac{R_{\lambda}}{R_{\lambda, 1}^{n_{\lambda,+1}}}=M_{\lambda, \lambda+1} \frac{R_{\lambda}}{R_{\lambda+1}^{n_{\lambda, \lambda+1}}} \\
\operatorname{TISI}_{\lambda, \lambda+1}=\left(\frac{T_{S, \lambda}}{T_{S, \lambda+1}}\right)^{n_{\lambda}}
\end{gathered}
$$

Outro índice pode ser obtido a partir da equação 23 , também independente da temperatura: 


$$
\operatorname{TISIE}_{\lambda, \lambda+1}=\frac{\varepsilon_{\lambda}}{\varepsilon_{\lambda+1}^{n_{\lambda,+1}}}
$$

Estes índices, até o momento, rejeitaram a contribuição da atmosfera. Considerando esta contribuição é necessário acrescentar um fator de correção na equação 22 :

$$
\begin{aligned}
& R_{\lambda}=\varepsilon_{\lambda} \alpha_{\lambda}\left(T_{0}\right) T^{n_{\lambda}\left(T_{0}\right)} C_{\lambda} \\
& T_{0}^{n_{\lambda}\left(I_{0}\right)}=\varepsilon_{\lambda} T_{S}^{n(\lambda, T)} C_{\lambda}
\end{aligned}
$$

com:

$$
C_{\lambda}=\frac{1-\beta_{\lambda}}{1-\left(R_{\lambda}^{\downarrow} / R_{\lambda}\right)} \text { e } \beta_{\lambda}=\frac{R_{\lambda}^{\downarrow}}{B_{\lambda}\left(T_{S}\right)}
$$

Com o fator de correção inserido o sistema completo pode ser expresso por:

$$
\begin{aligned}
& \operatorname{TISI}_{\lambda, \lambda+1}=\frac{C_{\lambda, \lambda+1} \operatorname{TISIE}_{\lambda, \lambda+1}}{M_{\lambda, \lambda+1}} \\
& \operatorname{TISI}_{\lambda, \lambda+1}=C_{\lambda, \lambda+1} \operatorname{TISIE}_{\lambda, \lambda+1}
\end{aligned}
$$

onde $C_{\lambda, \lambda+1}=C_{\lambda} / C_{\lambda+1}^{n_{\lambda, \lambda+1}}$. Para calcular o termo $C_{\lambda, \lambda+1}$ em função da temperatura real $\left(T_{r}\right)$, esta que é uma função da radiância de $R(\lambda, T)$ e $\beta_{\lambda}$, uma boa aproximação é substituir $B_{\lambda}\left(T_{r}\right)$ por $B_{\lambda}\left(\max \left(T_{S}\right)\right)$, com $\max \left(T_{S}\right)$ sendo a maior temperatura de brilho da superfície encontrada entre as bandas para um determinado pixel.

A partir deste índice é possível calcular a emissividade espectral, desde que se conheça a radiância atmosférica descendente em todas as bandas, e a emissividade em uma banda de referência $\left(\varepsilon_{\mathrm{r}}\right)($ Becker e Li, 1990). O índice TISI entre uma banda qualquer e esta banda de referência é dado por:

$$
\varepsilon_{\lambda}=\varepsilon_{r}^{n_{\lambda, r}} \frac{\operatorname{TISI}_{\lambda, r}}{C_{\lambda, r}}
$$

aonde:

$$
C_{\hat{\lambda}, r}=\frac{1-\beta_{\lambda}}{\left(1+\left(\frac{1-\varepsilon_{r}}{\varepsilon_{r}}\right) \beta_{r}\right)^{n_{\lambda,}}-\frac{\beta_{\lambda}}{T I S I_{\lambda, r} \varepsilon_{r}^{n_{2,}}}}
$$

\subsection{Método da Banda de Referência (MBR)}

Kahle et al., (1980) desenvolveram este método onde se assume que determinada banda (banda $r$ por exemplo)tem emissividade constante para todos os pixels. A seleção desta banda está condicionada ao conhecimento prévio do alvo em análise e sua emissividade. Conhecendo os 
parâmetros atmosféricos $\left(\tau_{r}, R_{r}^{\downarrow}\right.$ e $\left.R_{r}^{\uparrow}\right)$ desta banda é possível calcular uma aproximação da temperatura de superfície $\left(T_{s r}\right)$ para cada pixel a partir das medidas de radiância $I_{r}$ utilizando a inversa das equações 5 e 6 :

$$
T_{s r}=B_{r}^{-1}\left(\frac{I_{r}-R_{r}^{\uparrow}-\left(1-\varepsilon_{r}\right) R_{r}^{\downarrow} \tau_{r}}{\varepsilon_{r} \tau_{r}}\right)
$$

Essa temperatura é então usada nas equações 5 e 6 para obter as emissividades dos pixels nas demais bandas, conforme:

$$
\varepsilon_{r}=\frac{I_{r}-R_{r}^{\uparrow}-\tau_{r} R_{r}^{\downarrow}}{\tau_{r}\left(B_{r}\left(T_{s r}\right)-R_{r}^{\downarrow}\right)}
$$

\subsection{Método da Emissividade Normalizada (MEN)}

Desenvolvido por Gillespie (1985), e sendo mais sofisticado que o MBR (Gillespie et al., 1999), assume-se um único valor para a emissividade máxima em todas as $N$ bandas, para todos os pixels desta banda. Assim, a partir das radiâncias medidas, de uma emissividade de referência, e da equação 31 são obtidas $N$ temperaturas para cada pixel. Em cada pixel, seleciona-se a maior dentre essas temperaturas como sendo a temperatura de brilho da superfície, e esta temperatura é então utilizada para calcular a nova emissividade para todas as bandas. O cálculo das demais emissividades é realizado como no MBR. As temperaturas são calculadas conforme:

$$
T_{\lambda, i}=B_{\lambda}^{-1}\left(\frac{I_{\lambda, i}-R_{\lambda}^{\hat{\imath}}-\left(1-\varepsilon_{r}\right) R_{\lambda}^{\downarrow} \tau_{\hat{\lambda}}}{\varepsilon_{r} \tau_{\lambda}}\right)
$$

aonde $\varepsilon_{\mathrm{r}}$ é a emissividade de referência, $I_{\lambda, i}$ é a radiância medida na banda $\lambda$ para o píxel $i$, os termos $R_{\lambda}^{\uparrow}, R_{\lambda}^{\downarrow}$ e $\tau_{\lambda}$ são, respectivamente, a radiância ascendente, a radiância descendente e $\tau$ é a transmissividade atmosférica para o comprimento de onda $\lambda$. A emissividade para o pixel $i$ é calculada pela equação:

$$
\varepsilon_{\lambda, i}=\frac{I_{\lambda, i}-R_{\lambda}^{\uparrow}-\tau_{\lambda} R_{\lambda}^{\downarrow}}{\tau_{\lambda}\left(B_{\lambda}\left(T_{\max , i}\right)-R_{\lambda}^{\downarrow}\right)}
$$

com $T_{\max , i}$ sendo a temperatura máxima dentre as temperaturas calculadas para o pixel $i$.

\subsection{Método dos Resíduos Alpha ( $\alpha-R M)$}

O método inicialmente foi proposto por Green e Craig (1985) sob o nome de Resíduos Logarítmicos (RL), no entanto tem sido modificado por diversos autores desde então de modo a 
eliminar restrições e erros na proposta inicial. Hook et al., (1992) propuseram a forma final do RL. No entanto, este método ainda é imagem dependente, ou seja, depende de informações da cena ou da região analisada. O RL utiliza a aproximação de Wien para a função de Planck, dada por:

$$
\begin{aligned}
\lambda \ln [I(\lambda)]=\lambda \ln [\varepsilon(\lambda, T)]+\lambda \ln \left(C_{1}\right) & \\
& -5 \lambda \ln (\lambda)-\lambda \ln (\pi)-\frac{C_{2}}{T}
\end{aligned}
$$

A equação 33 para temperaturas de $\approx 300 \mathrm{~K}$ e comprimentos de onda $\geq 10 \mu \mathrm{m}$ tem erro máximo de 1\% (Siegel e Howel, 1982; Grondona et al., 2013).

O $\alpha$-RM é uma simplificação melhorada do RL (Hook et al., 1992; Kealy e Hook, 1993 e Gillespie et al., 1999), com a imagem dependência eliminada:

$$
\alpha_{j, \lambda}=X_{j, \lambda}-X_{j, M}
$$

onde $j$ é o pixel em análise, $X_{j, \lambda}$ é o logaritmo ponderado da radiância, $X_{j, M}$ é a média da radiância sobre todas as bandas para o pixel $j$, expressos por:

$$
\begin{gathered}
X_{j, \lambda}=\lambda \ln \left(\varepsilon_{j, \lambda}\right) \\
X_{j, \mathrm{M}}=\frac{1}{D} \sum_{i=1}^{D} \lambda_{i} \ln \left(\varepsilon_{j, \lambda_{i}}\right)
\end{gathered}
$$

com $D$ sendo o número de bandas. Assim, o cálculo dos resíduos alpha $\left(\alpha_{j, \lambda}\right)$ é a diferença entre a média da radiância sobre todas as bandas para o pixel $j$ e a equação linearizada da radiância (equação 33). Desta forma, um conjunto de $i$ equações são obtidas, e tem a seguinte forma:

$$
\begin{aligned}
& \lambda \ln \left(\varepsilon_{j, \lambda}\right)-\mu_{j, \alpha_{i}}=\alpha_{j, \lambda}= \\
& \lambda \ln \left(I_{j, \lambda}\right)-\frac{1}{D} \sum_{i=1}^{D} \lambda_{i} \ln \left(I_{j, \lambda_{2}}\right)+K_{\lambda}
\end{aligned}
$$

com $\mu_{j, \alpha_{\lambda}}$ e $K_{\lambda}$ sendo:

$$
\begin{gathered}
\mu_{j, \alpha_{2}}=\frac{1}{D} \sum_{i=1}^{D} \lambda_{i} \ln \left(\varepsilon_{j, \lambda_{i}}\right) \\
K_{\hat{\lambda}=5}=5 \ln (\lambda)-\frac{5}{D} \sum_{i=1}^{D} \lambda_{i} \ln \left(\lambda_{i}\right) \\
-\left(\ln \left(C_{1}\right)-\ln (\pi)\right)\left(\lambda-\frac{1}{D} \sum_{i=1}^{D} \lambda_{i}\right)
\end{gathered}
$$

Deve-se notar que o termo $K_{\lambda}$ provém apenas de parâmetros do sensor, e é independente da radiância.

Os resíduos $\alpha_{j, \lambda_{i}}$ são calculados somente com base na radiância medida, através do lado direito da equação 37, e o seu espectro é proporcional à emissividade. Embora a dependência da temperatura tenha sido eliminada, sendo substituída pelo termo $\mu_{j, \alpha_{\lambda_{i}}}$ o número de variáveis desconhecidas não muda, e para calcular a emissividade a partir de $\alpha_{j, \lambda_{i}}$ é necessário estimar o 
termo $\mu_{j, \alpha_{\lambda_{i}}}$. Isto pode ser realizado através de uma regressão na variância de $\alpha_{\lambda_{i}}$ para os dados de laboratório:

$$
\mu_{j, \alpha_{i}}=c\left(\frac{1}{D-1} \sum_{i=1}^{D} \alpha_{i}^{2}\right)^{(1 / x)}
$$

com $c$ e $x$ sendo coeficientes determinados empiricamente (Gillespie et al., 1999). O espectro de emissividade pode ser obtido invertendo a equação 37 :

$$
\varepsilon_{j, \lambda_{i}}=\exp \left(\frac{\alpha_{j, \lambda_{i}}+\mu_{j, \alpha_{z_{i}}}}{\lambda_{i}}\right)
$$

\subsection{Método de Máxima-Mínima Diferença (MMD)}

Proposto por Matsunaga (1994), usa a relação que existe entre a emissividade média $(\bar{\varepsilon})$ e a diferença entre sua variação máxima e mínima. Partindo do espectro médio de emissividade, e utilizando um processo iterativo, a emissividade média é re-estimada de acordo com a diferença entre o valor máximo e mínimo da emissividade na iteração anterior. No final do processo, a temperatura é finalmente calculada. Este processo pode ser descrito em 5 passos (Matsunaga, 1994; Gillespie et al., 1999; Coll et al., 2007):

$\left.1^{\circ}\right)$ Estimativa inicial do espectro de emissividade, geralmente com o MEN;

$\left.2^{\circ}\right)$ O MMD é calculado a partir do passo anterior para a primeira iteração, nas demais o MMD é calculado a partir dos espectros de emissividades ajustados, conforme:

$$
M M D_{\lambda, t}=\max \left(\varepsilon_{\lambda, t}\right)-\min \left(\varepsilon_{\lambda, t}\right)
$$

onde $\max \left(\varepsilon_{\lambda, t}\right)$ e $\min \left(\varepsilon_{\lambda, t}\right)$ são, respectivamente, a emissividade máxima e mínima para a banda $\lambda$, na iteração $t$.

$3^{\circ}$ ) A nova emissividade média, $\bar{\varepsilon}_{t+1}$, é calculada utilizando a expressão:

$$
\bar{\varepsilon}_{t+1}=a+b *\left(M M D_{t}\right)
$$

Com $a$ e $b$ sendo constantes determinadas por meio de regressão em um conjunto de espectros de laboratório para vários alvos. O termo a pode ser interpretado como sendo a emissividade dos alvos com comportamento semelhante a um corpo cinza (água, neve e vegetação), podendo, estes termos, serem aplicados em um amplo conjunto de dados sem que haja a necessidade de uma nova estimativa;

$\left.4^{\circ}\right)$ O ajuste das emissividades é realizado conforme a equação:

$$
\varepsilon_{\lambda, t+1}=\varepsilon_{\lambda, t} *\left(\frac{\bar{\varepsilon}_{t+1}}{\bar{\varepsilon}_{t}}\right)
$$

$\left.5^{\circ}\right)$ A temperatura é calculada a partir de $\varepsilon_{\lambda, t+1}$ e a sua respectiva radiância; 
O processo iterativo prossegue do passo 2 ao passo 5, até que a diferença da temperatura entre as iterações seja menor que o um dado limiar $(N E \Delta T)$, pré-estabelecido, de modo que $T_{t+1}-T_{t}<N E \Delta T$.

\subsection{Método do Corpo Cinza (MCC)}

Proposto por Barducci e Pippi (1996) é baseado na hipótese de que o espectro de emissividade do alvo em questão é plano o suficiente de modo que a variação da emissividade é desprezível, ou seja, a emissividade varia pouco com relação ao comprimento de onda.Esta hipótese é muitas vezes verificada, principalmente nos espectros de emissividade obtidos em laboratório, ou a partir de sensores termais hiperespectrais, e pode ser escrita matematicamente como:

$$
\begin{gathered}
\frac{d \varepsilon}{d \lambda}=0 \\
\varepsilon\left(\lambda_{i}\right)=\varepsilon\left(\lambda_{j}\right)
\end{gathered}
$$

com $\varepsilon\left(\lambda_{k}\right)$ sendo a emissividade média para a banda $k(k=1, \ldots, N)$.

As hipóteses das equações 45 e 46 são conceitualmente iguais, porém, cada uma se aplica em uma determinada situação. Enquanto a primeira serve para aplicações sobre o espectro contínuo, a segunda se aplica a intervalos do espectro, ou seja, a hipótese da equação 45 é mais geral que a hipótese da equação 46.

O algoritmo para o MCC parte da reescrita da radiância, como:

$$
R_{k}=\varepsilon\left(\lambda_{k}, T\right) * B\left(\lambda_{k}, T\right)+n_{k}
$$

onde $n_{k}$ é um termo aditivo com média zero, referente ao ruído. Em seguida o algoritmo procura por estimativas para temperatura $T$ e emissividade $\varepsilon$ que minimizam o erro $E$ :

$$
E=\sum_{k=1}^{N}\left[R_{k}-e\left(\lambda_{k}, t\right) * B\left(\lambda_{k}, t\right)\right]^{2}
$$

Vale notar que $\varepsilon$ e $T$ representam os valores reais de emissividade e temperatura do alvo, enquanto $e$ e $t$ são, respectivamente, as estimativas destes valores. Utilizando uma estrutura iterativa para a minimização, a expressão geral para os termos $e$ e $t$ pode ser expressa por:

$$
\begin{aligned}
& e_{i}\left(\lambda_{k}, t_{i}\right)=\frac{\sum_{k=1}^{N}\left[B\left(\lambda_{k}, t_{i}\right) * R_{k}\right]}{\sum_{k=1}^{N}\left[B^{2}\left(\lambda_{k}, t_{i}\right)\right]} \\
& t_{i+1}=t_{i}+\delta t_{i}
\end{aligned}
$$




$$
\delta t_{i}=-\frac{\left(\frac{d E\left(t_{i}\right)}{d t}\right)}{\left(\frac{d^{2} E\left(t_{i}\right)}{d t^{2}}\right)}
$$

com $i$ sendo a iteração. No processo iterativo uma temperatura inicial $\left(t_{0}\right)$ é assumida, e a primeira estimativa para a emissividade $\left(e_{0}\right)$ é calculada. Em seguida, a temperatura a ser utilizada na próxima iteração é calculada, e uma nova estimativa para a emissividade é feita, e assim sucessivamente. $O$ processo itera até que a temperatura calculada seja minimize a equação 48, ou seja:

$$
\frac{d E\left(t=t_{i}\right)}{d t}=0
$$

\section{Resultados e Discussões}

Cada um dos nove métodos analisados possuí propriedades matemáticas e suposições de aplicabilidade que estão diretamente relacionadas com a qualidade e a precisão do resultado final. É de suma importância entender tais limitações para uma escolha adequada do método a ser utilizado.

O RCD permite que uma composição falsa-cor (RGB) mostre diferenças de emissividade como variações nas cores, enquanto diferenças na temperatura são mostradas como variações na intensidade. Este método não retorna os valores para temperatura/emissividade, pois mostra somente a sua variação. Além disso, o método é imagem-dependente, ou seja, o resultado de usar a cena inteira é diferente do resultado obtido utilizando somente um recorte da cena inicial. Se houver grandes variações na temperatura dentro da imagem a informação pode ser degradada, pois áreas frias aparecem escuras mascarando leves variações na cor (variações na emissividade). O RE tem pouca sensibilidade a variações na temperatura, bastando somente uma aproximação da temperatura para obter razões com erro menor que $1 \%$. É aplicável,portanto,a qualquer sensor que adquira dados com mais de uma banda espectral. Os resultados necessitam de um processamento adicional para comparação das curvas espectrais. Em geral faz-se necessário converter os dados de laboratório em razões para tal comparação. Devido à razão utilizada na formulação, este método também realça as altas freqüências.

O MDT calcula diretamente a emissividade dos dados, sem a necessidade de suposições iniciais quanto à forma da curva espectral do alvo. Combinado com o RSpoder ser utilizado na exploração mineral. Neste método é necessário assumir que a emissividade é invariante no tempo, e implicitamente que a emissividade não muda com a variação na temperatura. Esta hipótese é aceitável, desde que a variação entre as temperaturas não seja significativamente grande. Entretanto, o método ainda não está bem estabelecido para a vegetação, e é definitivamente inválida para mudanças transitórias (e.g. mistura dos solos, nos materiais superficiais, chuvas ou outros eventos entre as medições). Outraspossíveis fontes de erro são o registro das imagens e a possível existência de ruído devido a razão existente (i.e. solução da equação 15). 
O TISI é praticamente independente da temperatura, as razões TISI e TISIE são próximas, e conforme a temperatura aumenta, esta diferença diminui. A diferença entre esses índices é da ordem de $1 \%$, e pode ser ainda menor com emissividades maiores e melhores condições atmosféricas. O TISI é facilmente calculável diretamente dos dados de radiância, sem a necessidade de informações a priori sobre a emissividade/temperatura. Entretanto, é muito sensível a variações das características da superfície. Conforme Becker e Li (1990) este índice pode ser até 12 vezes mais preciso que uma simples razão espectral. Porém, sem a correção atmosférica, ou havendo problemas, este erro é propagado para o TISI . Além disso, é necessário uma emissividadede referência para o cálculo da emissividade nas demais bandas, e o sensor deve ter no mínimo duas bandas termais (facilmente aplicável a mais de duas bandas simultaneamente). Assim, índicesmais adequados a uma aplicação podem existir, dependendo da combinação de bandas utilizadas (Becker e Li, 1990). Por último, o índice não é muito sensível às características da vegetação, ao contrário de solos expostos.

O MBR teve grande importância para pesquisas com TIR, sendo também chamado, por este motivo, de método modelo de emissividade (Gillespie et al., 1999). Conforme conhecimento $a$ priori do alvo, o valor máximo para a emissividade e a banda podem ser selecionados. Para os silicatos esta banda está sempre localizada nos maiores comprimentos de onda do TIR, e a emissividade máxima é de $\approx 0.95$. O problema deste método está em assumir que todos os pixels da imagem, em uma determinada banda espectral, tem a mesma emissividade. Alvos como vegetação, neve e água teriam a temperatura subestimada, pois a sua emissividade é maior do que a proposta anteriormente. Assim, este método não produz resultados acurados, simultaneamente, para alvos com emissividades muito diferentes.

No MEN, a emissividade máxima ocorre na banda que tiver a temperatura máxima para o pixel em questão. Além disso, este método herda todas as vantagens do RCM, e é menos propenso a erros. A acurácia do NEM depende do valor inicial da emissividade máxima utilizado, porém, é incapaz de produzir bons resultados, simultaneamente, para alvos com emissividades muito diferentes. Se houver um alvo com emissividade maior do que a emissividade máxima, inicialmente definida, a temperatura para este alvo será subestimada.

No $\alpha$-RM a comparação dos dados (laboratório-campo) não é direta, sendo necessária a conversão para a forma de resíduos alpha ou para emissividade conforme a equação 41. A principal vantagem deste método é a sua independência em relação à temperatura, com pouca propagação de ruído de uma banda para as demais. Além disso, geram-se bons resultados para diferentes alvos simultaneamente, é imagem-independente e preserva a forma da curva espectral da emissividade, mas não a sua amplitude. Por último, outra desvantagem é a utilização de uma aproximação para a função de Planck (aproximação de Wien), mas desde que sejam observadas as restrições os erros são inferiores a 1\% (Grondona et al., 2013).

No MMD a temperatura é relativamente insensível ao espalhamento múltiplo da radiação termal e a radiância descendente, dentro de um elemento da cena, porém as emissividades calculadas são mais sensíveis a esses fatores. A acurácia do MMD e do NEM são similares, mas no MMD depende da precisão da relação entre $\bar{\varepsilon}$ e o $M M D$ e/ou $N E \Delta T$. O erro quadrático médio do MMD é menor que o do NEM, exceto quando $N E \Delta T>0.3 \mathrm{~K}$. No geral, o MMD é mais preciso, e mais sensível a erros de medida, que o NEM.

O MCC é capaz de obter a temperatura e a emissividade de um alvo sem nenhum conhecimento sobre o valor real da emissividade/temperatura. Para sensores hiperespectrais o algoritmo se mostra promissor, pois a hipótese dada pela equação 45 é mais facilmente verificada. No entanto, a maioria dos alvos da cobertura terrestre não satisfaz esta hipótese, principalmente em sensores multiespectrais, devido ao seu pequeno número de bandas no termal (Gillespie et al., 1999). O método é sensível a erros de medida, principalmente quando se utiliza a hipótese da equação 46, onde a temperatura e a emissividade podem ser calculados diretamente através da razão de bandas. 
Deve-se salientar que uma fonte de erros comum a todos os métodos, e que deve ser levada em conta, são as áreas com nuvens. Apesar dos comprimentos de onda do TIR serem menos sensíveis a interferências causadas pelas nuvens do que as regiões do visível e do infravermelho próximo, este alvo não é invisível no TIR, e os métodos analisados não se aplicam em tais áreas. A nuvem nada mais é do que vapor de água, que por sua vez é o maior causador de problemas no TIR, e a aplicação de métodos de filtragem de nuvens degrada os dados uma vez que não se tem o valor real de reflectância/radiância dos alvos abaixo das nuvens, e sim uma estimativa do mesmo. Também deve-se considerar que a resolução espacial pode ser um problema, porém este problema está relacionado principalmente a escala do estudo, e tem pouca relação com o método escolhido.

Nas Tabelas 1 e 2 as restrições e aplicações, respectivamente, de cada um dos métodos podem ser vistas de forma resumida, de modo a favorecer a comparação e escolha do método mais adequado ao problema.

Tabela 1: Restrições dos métodos

\begin{tabular}{|c|c|c|c|c|c|c|c|c|c|}
\hline & $\mathrm{RE}$ & MDT & TISI & MBR & MEN & $a-R M$ & MMD & MCC & RCD \\
\hline $\begin{array}{c}\text { Conhecimento prévio do } \\
\text { alvo }\end{array}$ & $\mathrm{x}$ & & & $\mathrm{x}$ & $\mathrm{x}$ & & $\mathrm{x}$ & & \\
\hline $\begin{array}{l}\text { Proc. de vários alvos } \\
\text { simult. }\end{array}$ & $\mathbf{x}$ & $\mathrm{x}$ & $\mathrm{x}$ & & & $\mathrm{x}$ & $\mathrm{x}$ & $\mathrm{x}$ & $\mathrm{x}$ \\
\hline $\begin{array}{l}\text { Correção atmosférica } \\
\text { indispensável }\end{array}$ & & & $\mathrm{x}$ & & & $\mathrm{x}$ & $\mathrm{x}$ & & \\
\hline $\begin{array}{l}\text { Comp. direta dos } \\
\text { resultados/curva } \\
\text { emissividade }\end{array}$ & & $\mathrm{x}$ & & $\mathrm{x}$ & $\mathrm{x}$ & & $\mathbf{x}$ & $\mathrm{x}$ & \\
\hline Emissividade & $\mathrm{R}$ & $\mathrm{A}$ & $\mathrm{R}$ & A & $\mathrm{A}$ & $\mathrm{R}$ & $\mathrm{R}$ & A & Não calcula \\
\hline
\end{tabular}

$* \mathrm{R}=$ emissividade relativa $/ \mathrm{A}=$ Emissividade Absoluta

Tabela 2 : Aplicações dos métodos

\begin{tabular}{c|c|c}
\hline Observações & Aplicação \\
\hline RE & $\begin{array}{c}\text { O erro na razão das emissividade é menor que o erro } \\
\text { na emissividade em si }\end{array}$ & Estudo da variação de emissividade \\
\hline MDT & $\begin{array}{c}\text { Assume-se que a emissividade é invariante no tempo } \\
\text { (temperatura) e é necessário fazer o registro das } \\
\text { imagens; }\end{array}$ & $\begin{array}{c}\text { Exploração mineral, podendo ser aplicado em } \\
\text { estudos de vegetação; }\end{array}$ \\
\hline TISI & $\begin{array}{c}\text { O indice é praticamente independente da temperatura, } \\
\text { e é muito sensivel a variações na composição do alvo }\end{array}$ & $\begin{array}{c}\text { Estudos de variação e emissividade } \\
\text { principalmente com solos, não tem boa resposta } \\
\text { para a vegetação }\end{array}$ \\
\hline MBR & Todos os alvos da cena tem mesma emissividade & Cálculo da temperatura \\
\hline MEN & Os resultados são mais precisos que o MBR & Cálculo da temperatura e emissividade \\
\hline MMD & $\begin{array}{c}\text { Tem resultados mais precisos que o LRM } \\
\text { partir de medidas de laboratório de emissividade para } \\
\text { um conjunto de alvos }\end{array}$ & $\begin{array}{c}\text { Estudos da forma da curva espectral de } \\
\text { emissividade, podendo-se estimar a } \\
\text { emissividade espectral }\end{array}$ \\
\hline MCC & $\begin{array}{c}\text { São necessárias no mínimo duas bandas, } \\
\text { preferencialmente não muito distantes }\end{array}$ & $\begin{array}{c}\text { Estimativas de temperatura e emissividade, } \\
\text { principalmente em sensores hiperespectrais e } \\
\text { dados de laboratório }\end{array}$ \\
\hline RCD & $\begin{array}{c}\text { Os resultados não separam a temperatura da } \\
\text { emissividade, além disso, pode haver perda de } \\
\text { informações em dados com grande variação na } \\
\text { temperatura }\end{array}$ & $\begin{array}{c}\text { Análise (visual) de variações de temperatura e } \\
\text { emissividade }\end{array}$ \\
\hline
\end{tabular}




\section{Conclusão}

Todos os métodos descritos anteriormente representam a maior, e principal, parte dos métodos existentes de separação de temperatura e emissividade desenvolvidos nas últimas quatro décadas. Outros métodos, mais recentes, são propostos, porém são aplicados em casos muito específicos de sensores, alvos ou determinados estudos e não fazem parte do objetivo deste trabalho. Os métodos abordados neste trabalho, apesar de serem aplicáveis em várias situações, produzem dados mais precisos se aplicados de acordo com suas restrições/limitações. Todos estes métodos foram desenvolvidos com base em dados de laboratório, por isso, deve-se salientar que todos requerem a correção atmosférica dos dados. Alguns são mais sensíveis aos erros desta correção (se não realizada corretamente), outros são mais robustos. Os métodos mais sensíveis são todos aqueles que (em algum momento) possuem uma razão de bandas espectrais, ou algum tipo de manipulação algébrica que posa realçar o ruído, dentre estes métodos podemos citar: RE, MDT, TISI e o $\alpha$-RM.

Outro ponto importante é que a maioria dos métodos foram desenvolvidos para o estudo de solos e rochas, uma vez que o TIR é voltado para esse tipo de estudo, e a vegetação tem pouca diferenciação nesta região do espectro eletromagnético. Apesar disso, alguns destes métodos podem retornar informações para estudos de vegetação.

Todos os métodos que necessitam de duas ou mais imagens da mesma área estão propensos a erros de registro e georreferenciamento, o que pode ser uma tarefa ainda mais complicada no caso de dados de sensores aerotransportados. Pode-se concluir também que todos os métodos são implementáveis, de baixo custo computacional, e de rápido processamento, e um resumo de suas aplicações, restrições e observações das particularidades podem ser vistos nas Tabelas 1 e 2 .

De modo geral, o MEN é o que produz melhores resultados quando se tem o conhecimento $a$ priori da emissividade máxima do alvo para qualquer tipo de estudo, o TISI e o $\alpha$-RM geram boas estimativas para a emissividade relativa de alvos (vegetação e solo) a partir de uma emissividade de referência sem o conhecimento da temperatura e o MCC é o mais indicado para sensores hiperespectrais e alvos que tenham pouca variação na emissividade para duas bandas adjacentes.

Estes métodos cumprem de forma satisfatória uma ampla gama de aplicações, no entanto, devido aos avanços tecnológicos nos sensores e a necessidade de estudos/monitoramento mais precisos da superfície terrestre se faz necessário pesquisas em métodos mais precisos e menos suscetíveis a erros na separação da temperatura e emissividade dos alvos.

\section{AGRADECIMENTOS}

Os autores gostariam de agradecer a Fundação de Amparo à Pesquisa do Estado do Rio Grande do Sul (FAPERGS) pela bolsa de estudos e ao Laboratório de Sensoriamento Remoto Geológico (LabSRGeo) do Centro Estadual de Pesquisa em Sensoriamento Remoto e Meteorologia (CEPSRM) da UFRGS.

\section{REFERÊNCIAS BIBLIOGRÁFICAS}

Barducci, A. and Pippi, I. "Temperature and emissivity retrieval from remotely sensed images using the "Grey body emissivity" method" IEEE Transactions on Geoscience and Remote Sensing, v.34, n.3, p. 681-695, 1996. 
Becker, F. and Li, Z. "Temperature-Independent Spectral Indices in Thermal Infrared Bands" Remote Sensing of Environment, v.32, p. 17- 33, 1990.

Becker, F. and Li, Z. "Surface Temperature and Emissivity at Various Scales: Definition, Measurement and Related Problems" Remote Sensing Reviews, v. 12, p. 225-253, 1995.

Coll, C., Caselles, V., Valor, E., Niclòs, R., Sánchez, J. M., Galve, J. M. and Mira, M. "Temperature and Emissivity Separation from ASTER Data for Low Spectral Contrast Surface" Remote Sensing of the Environment, v. 110, p. 162-175, 2007.

Collins, E. F., Roberts, D. A., Sutton, P. C., Funk, C. C., and Borel, C. C. "Temperature Estimation and Compositional Mapping Using Spectral Mixture Analysis of Thermal Imaging Spectrometry Data" SPIE Conference on Imaging Spectrometry, 1999.

Collins, E. F., Roberts, D.A. and Borel, C.C. "Spectral Mixture Analysis of Simulated Thermal Infrared Spectrometry Data: An Initial Temperature Estimate Bounded TESSMA Search Approach" IEEE Transactions on Geoscience and Remote Sensing, v.39, no.7, p. 1435-1446, 2001.

Gillespie, A. R. "Lithologicmapping of silicaterocksusingTIMS. The TIMS" Paper presented at the Data User's Workshop, JPL Publication, 1985, v. 86, n 38, p. 29-44.

Gillespie$^{1}$, A. R. "Enhancement of Multispectral Thermal Infrared Images: Decorrelation Contrast Stretching" Remote Sensing of the Environment, v. 42, p. 147-155, 1992.

Gillespie $^{2}$, A. R. "Spectral Mixture Analysis of Multispectral Thermal Infrared Images" Remote Sensing of the Environment, v. 42, p. 137-145, 1992.

Gillespie, A. R., Kahle, A. B. and Walker, R. E. "Color Enhancement of Highly Correlated Images. I. Decorrelation and HIS Contrast Stretches" Remote Sensing of the Environment, v. 20, p. 209-235, 1985.

Gillespie, A., Rokugawa, S., Matsunaga, T., Cothern, J. S., Hook, S. and Kahle, A. B. "Temperature/emissivity Separation Algorithm Theoretical Basis Document, version 2.4., 1999" Disponível online em: http://eospso.gsfc.nasa.gov/sites/default/files/atbd/atbd-ast-05-08.pdf (acessado 22 de novembro de 2013)

Green, A. A. and Craig, M. D. "Analysis of aircraft spectrometer data with logarithmic residuals" Paper presented at the Proceedings of the airborne imaging spectrometer data analysis workshop. Pasadena, CA. 1985, p. 111-119.

Grondona, A., Rolim, S. B. A., Ferreira, M. P., Veettil, B. K. and Iglesias, M. L. "Avaliação da propagação de erros ao utilizar a aproximação de Wien na linearização da lei de Planck" Anais XVI Simpósio Brasileiro de Sensoriamento Remoto - SBSR, Foz do Iguaçu, PR, Brasil, 13 a 18 de abril de 2013.

Hardy, J. D. and Muschemkeim, C. "The radiation of heat from the human body. IV. The emission, reflection, and transmission of infrared radiation by the human skin" Journal of Clinical Investigation, v.13, $\mathrm{n}^{\circ}$ 5, p. 817-831, 1934.

Hook, S. J., Gabell, A. R., Green, A. A. and Kealy, P. S. "A Comparison of Techniques for Extracting Emissivity Information from Thermal Infrared Data for Geologic Studies" Remote Sensing of the Environment, v. 42, p. 123-135, 1992.

Kahle, A. B. and Alley, R. E. "Separation of Temperature and Emittance in Remotely Sensed Radiance Measurements" Remote Sensing of the Environment, v. 42, p. 107-111, 1992.

Kahle, A. B., Madura, D. P. and Soha, J. M. "Middle infrared multispectral aircraft scanner data: analysis for geological applications" Applied Optics, v. 19, no. 14, p. 2279-2290, 1980. 
Kahle, A. B. and Rowan, L. C. "Evaluation of Multiespectral Middle Infrared Imagens for Lithologic Mapping in the East Tintic Mountains" Geology, v. 8, p.234-239, 1980.

Kealy, P. S. and Hook, S. J. "Separating Temperature and Emissivity in Thermal Infrared Multispectral Scanner Data: Implications for Recovering Land Surface Temperatures" IEEE Transactions on Geoscience and Remote Sensing, v.31, no.6, p. 1155-1164, 1993.

Li, Z., Becker, F., Stoll, M. P. and Wan, Z. "Evaluation of Six Methods for Extracting Relative Emissivity Spectra from Thermal Infrared Images" Remote Sensing of the Environment, v. 69, p. 197-214, 1999.

Matsunaga, T. A. "Temperature-Emissivity Separation Method Using an Empirical Relationship between the Mean, the Maximum, and the Minimum of the Thermal Infrared Emissivity Spectrum" Journal of Remote Sensing Soc. Japan, v. 14, no. 2, p. 230-241, 1994. (em japonês com resumo em inglês).

Schmugge, T., Hook, S.J. and Coll, C. "Recovering Surface Temperature and Emissivity from Thermal Infrared Multiespectral Data" Remote Sensing of Environment, v.65, p.121-131, 1998.

Siegel, R. and Howell, J. R. Thermal Radiation Heat Transfer. Second Edition, Hemisphere, New York, 1982.

Slater, P. N. Remote sensing, optics and optical system. Addison-Wesley, Reading, MA, 246247, 1980.

Sobrino, J. A. and Jimenez-Muñoz, J. C. "Surface emissivity retrieval from Digital Airborne Imaging Spectrometer data" Journal of Geophysical Research, v. 107, no. D23, 2002.

Soha, J. M. and Schwartz, A. A. "Multiespectral Histogram Normalization Contrast Enhancement" Proceedings 5th Canadian Symposium on Remote Sensing, Victoria, BC, Canada, 1978, p. 86-93.

Watson', K. "Spectral Ratio Method for Measuring Emissivity" Remote Sensing of the Environment, v. 42, p. 113-116, 1992.

Watson', K. "Two-Temperature Method for Measuring Emissivity" Remote Sensing of the Environment, v. 42, p. 117-121, 1992.

Recebido em fevereiro de 2015.

Aceito em outubro de 2015. 